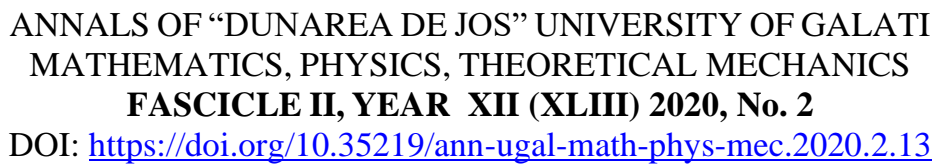

\title{
Evolutionary trends in oncological pathology in Galati and the purpose of environmental factors
}

\author{
Laura Rebegea ${ }^{1,2^{*}}$, Dorel Firescu ${ }^{3,4}$, Elena Niculeț ${ }^{5}$, Ana-Maria Pâslaru ${ }^{6}$, \\ Cristina Serban ${ }^{3,4}$, Mihaela Dumitru ${ }^{2}$ \\ ${ }^{1}$ Medical Clinical Department, "Dunărea de Jos" University, Faculty of Medicine and Pharmacy, Galați, \\ România. \\ ${ }^{2}$ Radiotherapy Department, Emergency Hospital "Sf Ap Andrei” Galați, România. \\ ${ }^{3}$ Surgical Clinical Department, "Dunărea de Jos" University, Faculty of Medicine and Pharmacy, Galați, \\ România \\ ${ }^{4}$ II ${ }^{\text {nd }}$ Surgery Clinic Emergency Hospital "Sf Ap Andrei"Galați, România. \\ ${ }^{5}$ Department of Morphological Sciences, "Dunărea de Jos" University, Faculty of Medicine and Pharmacy, \\ Galați, România \\ ${ }^{6}$ PhD-c Medical Department, "Dunărea de Jos" University, Faculty of Medicine and Pharmacy, Galați, \\ România. \\ Corresponding author: laura_rebegea@yahoo.com
}

\begin{abstract}
According to statistical and epidemiological data, the contemporary period is dominated by an increase in malignant tumors with various locations. Several epidemiological studies, both epidemiological and clinical have indicated as the cause of cancer a combination of multiple factors. It seems that there is a tight connection between the external environmental factors, some of which are considered carcinogens, the lifestyle of each individual, and endogenous factors. More than once, these factors have merged, so it becomes extremely difficult to highlight the contribution of each one of them in the process of neoplasia development.

This study is a retrospective one; it includes 8972 patients diagnosed with different kinds of neoplasia in the ambulatory specialty clinic at the Emergency Clinical Hospital of Galati between 2013 and 2019. Associated with this study there is an in-depth analysis of the current literature which was done from the following perspective: incidence, epidemiology, and etiology of cancer at a global level.

During the analyzed period, we observed a slight increase in the incidence of cases with cancer located in the prostate area, the breast, and colorectal. Malignant tumors of the breast registered the highest rate of incidence in 5 of the 7 years included in the study. In 2014 and 2018, the most frequent diagnosis was colorectal cancer. Although breast cancer was at the top of the incidence rates both in the first and the last years of the study, we noticed an increase in the number of cases in 2019 - 247, in comparison with 225 registered in 2013. As for sex distribution, the incidence was higher for male patients.

As a significant part of pathology, malignant tumors with various locations are an essential problem of public health, with a major impact on the population. Studying the environmental factors identified as being significantly responsible for the development of neoplasia, could allow categorizing the individuals exposed to pollution into risk groups.
\end{abstract}

Keywords: cancer, treatment, pollution 


\section{INTRODUCTION}

According to statistical and epidemiological data, the contemporary period is dominated by an increase in malignant tumors with various locations. Several epidemiological studies, both epidemiological and clinical have indicated as the cause of cancer a combination of multiple factors [1]. It would appear that there is a tight connection between the factors of the external environment, some of which carcinogens, the lifestyle of each individual, and endogenous factors [2,3]. More than a few times these factors merge so it becomes extremely difficult to tell the contribution of each one of them in the process of neoplasia development.

\section{MATERIAL AND METHOD}

The study is a retrospective one; it includes 8972 patients diagnosed with different kinds of neoplasia in the ambulatory section of specialty at the Clinical Hospital of Emergencies Galati between the period of time 2013-2019. Associated with this study, an analysis of the information in the literature of specialty was effectuated concerning the incidence, epidemiology, and etiology of cancer at a global level.

\section{RESULTS AND DISCUSSIONS}

Malignant tumors of the breast have registered the highest rate of incidence in the five years included in the study (Fig 1). Although mammary cancer was at the top of the incidence rates both in the first and the last years of the study we notice an increase in the numbers of cases in 2019 at 247 in comparison to 225 registered in 2013. The incidence of the cases of breast cancer has increased in 2019 by $105.83 \%$ in comparison to the cases in 2018 .

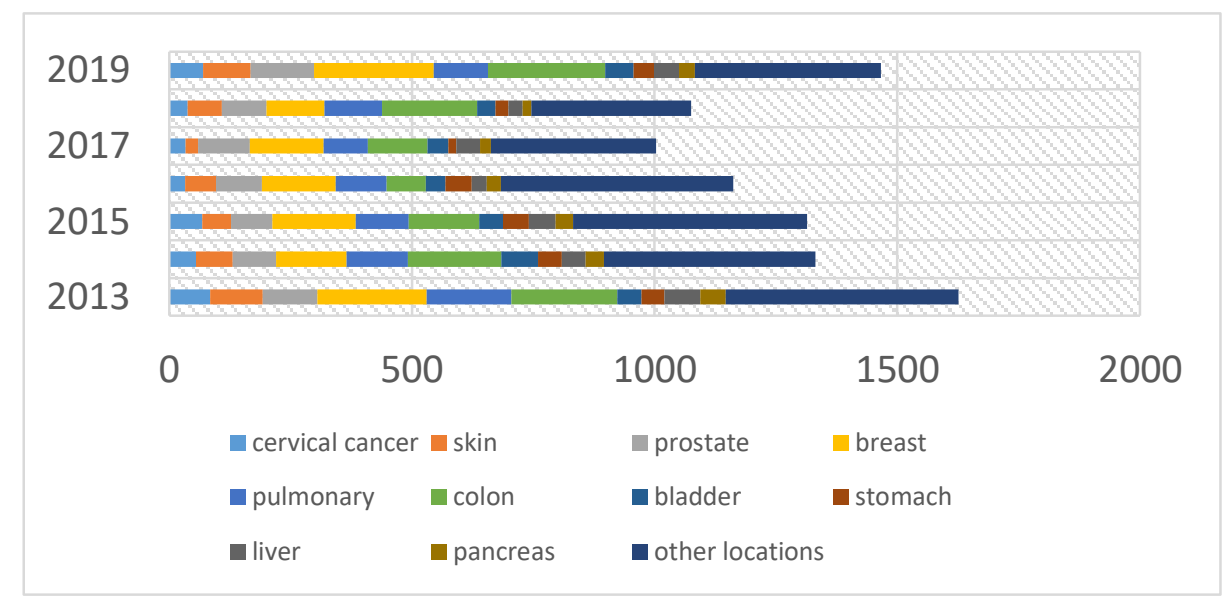

Fig 1. Frequent tumor location 2013-2019

In the time period 2013-2019, we observe a slight increase in the incidence of the cases, with locations in the prostate area, the breast, and colorectal. In 2014 and 2018 the most frequent diagnosis was colorectal cancer. The cases of colorectal malignant tumors have gradually increased, achieving a number of 241 new cases in comparison to the 196 cases registered in 2018.

In comparison to the former year, there was an increase in the total number of registered cases. In the period of the analysis, a slight decrease in the incidence of skin neoplasm was observed, 109 new cases were registered in 2013 while in 2019 the number was 98 . We mention that malignant melanoma was not included in the study, having a distinct classification. Hepatic and pancreatic tumors followed the same descending tendency (Fig. 2). 


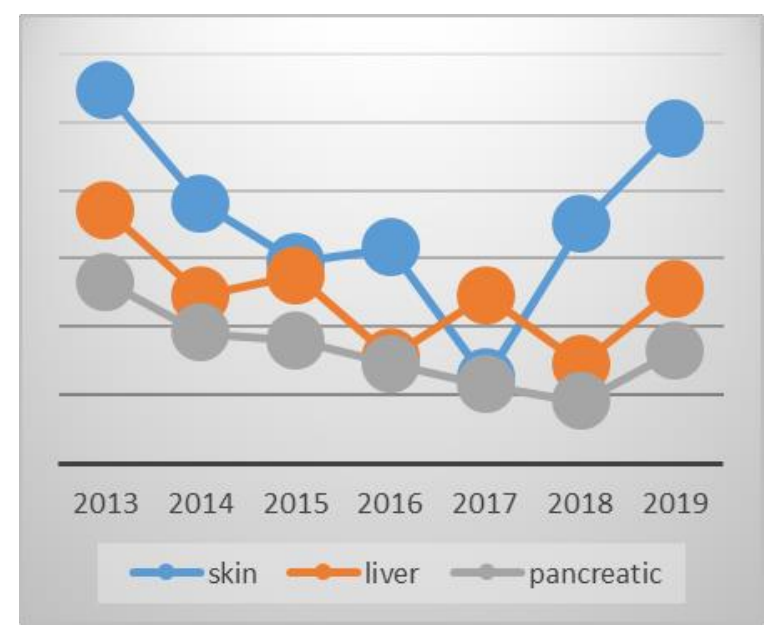

Fig. 2 Evolution of the incidence of malign tumors, skin, liver and pancreatic.

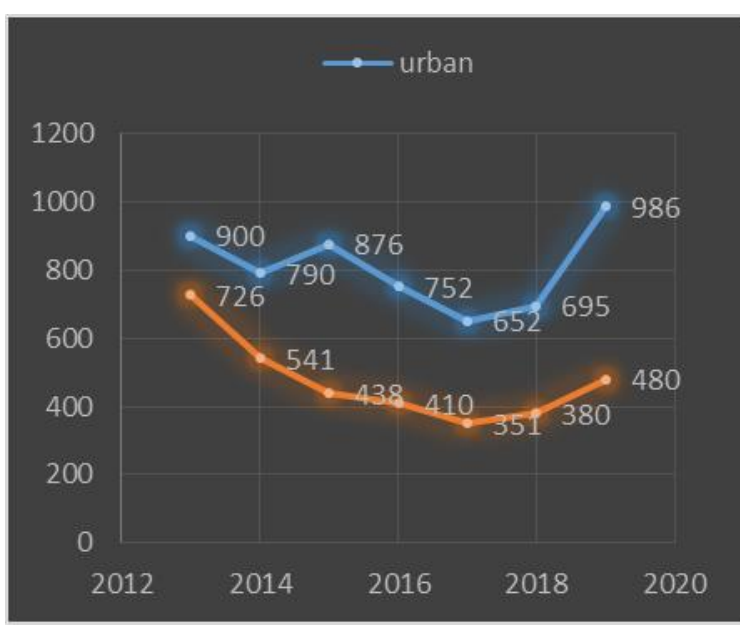

Fig. 3 Distribution of cases according to the environment of origin.

As for demographic distribution, the population from the urban area (5651) makes up most of the subjects analyzed for the study while the number of people from the countryside was that of 3326 . The results obtained are in conformity with the data from the specialty literature which appreciates a higher incidence rate amongst the population from urban areas [4,5]. This difference between urban areas and the countryside was most likely created by the difference of accessibility to medical checkups and information, accessibility which the patients from urban areas have. In the meantime, it is well known that patients from the countryside have limited knowledge of medical matters with very low accessibility to national programs of screening $[6,7,8]$. At the end of 2019, out of the total numbers of patients kept in evidence, $65 \%$ came from urban areas [9]. This percentage highlights the high rate of mortality amongst the patients from the countryside and also draws attention to the method of detecting neoplasia affections in advanced studies in which the treatment might more than often merely have a soothing effect and purpose.

At the same time, a parallel analysis was made which included follow-up patients of the oncological clinic, and as far as sex distribution concerns, 53\% (8761) of all patients are female patients. This highlights the mortality rate among male patients.

As a significant part of pathology, malignant tumors with various locations represent an essential problem for public health with a major impact on the population [10].

At a global level, the most frequent diagnosis of neoplasia is breast cancer amongst female patients [11], it is estimated that in 2020 the percentage of this affection to reach 13,3\% out of the total of cancer types diagnosed [12]. In our study, the results show up to be similar to international ones, in 2019 the percentage of mammary neoplasia being $16,84 \%$ out of the total of new cases diagnosed. Moreover, men can develop breast cancer, representing less than $1 \%$ of those diagnosed each year $[13,14]$. Amongst the risk factors responsible for the development of breast cancer amongst male patients are the following: exposure to radiation, high levels of estrogen, and family history with breast cancer may increase the risk of disease for a man. For men, the most frequent location is represented by prostate cancer [15].

The important demographic gap between the countryside and urban areas reflect the same tendency observed at a global level. In a report made in 2017 in America, realized with the purpose of identifying incidence lags and survival in cancer between the countryside and urban areas a lower incidence is observed amongst the population from the countryside but with a higher mortality rate than for the population in the urban areas [16]. The high incidence of the number of patients diagnosed with cancer might have been realized thanks to imagistic technology which has had a significant evolution in the last century which has allowed efficient diagnoses, the application of appropriate treatments, and the higher rate of survival which might be thanks to adopting an occidental-like lifestyle $[17,18]$. 
It seems that there is a strong link between the external environmental factors (some of which are cancerous agents), the lifestyle of each individual, and the endogenous factors. In most cases, these factors associate and interact with one another, being nearly impossible to differentiate each one's contribution to the process of neoplasia development.

\section{CONCLUSIONS}

Studying the causes of cancer helps researchers understand the carcinogenesis process and identify the risk factors of genetic type, environmental, and behavior for cancer. This knowledge may lead to new possibilities for preventing and treating the disease. Studying and researching the environmental factors identified as risk elements for the individual, the community or population, the mechanisms by which they can limit or facilitate a specific action on the human body and organism can be revealed.

The multi-disciplinary research teams are increasingly frequent and more common and often include a number of experts, epidemiologists, general medicine medics, calculation biologists, statisticians, oncologists, toxicologists and geneticists.

\section{References}

1 Sonnenschein C, Soto AM. Theories of carcinogenesis: an emerging perspective. Semin Cancer Biol. 2008;18(5):372-377.

2 Berrington de González A, Mahesh M, Kim KP, et al. Projected cancer risks from computed tomographic scans performed in the United States. Arch Intern Med. 2009;169(22):20712077.

3 Reimer KJ, Koch I, Cullen WR. Organo-arsenicals. Distribution and transformation in the environment. Metal Ions In Life Sciences. 2010;7:165-229.

4 Andriole GL, Bostwick DG, Brawley OW, et al. Effect of dutasteride on the risk of prostate cancer. N Engl J Med. 2010;362(13):1192-2202.

5 Andersson TM, Engholm G, Brink AL, Pukkala E, Stenbeck M, Tryggvadottir L, Weiderpass E, Storm H. Tackling the tobacco epidemic in the Nordic countries and lower cancer incidence by $1 / 5$ in a 30 -year period-The effect of envisaged scenarios changing smoking prevalence. Eur J Cancer. 2018; 29(18): 30239-9.

6 Huang T, Yang B, Zheng J, Wahlqvist ML, Li D. Cardiovascular disease mortality andcancer incidence in vegetarians: a meta-analysis and systematic review. Ann Nutr Metab. 2012; 60(4): 233-240,

7 Schulz M, Hoffmann K, Weikert C, Nöthlings U, Schulze MB, Boeing H. Identification of a dietary pattern characterized by high-fat food choices associated with increased risk of breast cancer: the European Prospective Investigation into Cancer and Nutrition (EPIC)-Potsdam Study. Br J Nutr. 2008; 100(5): 942-946

8 Szmigielski S. Risk of neoplastic diseases in conditions of exposure to power magnetic fields - epidemiological investigations. Med Pr. 2009; 60(3): 223-233.

9 Szmigielski S. Risk of neoplastic diseases in conditions of exposure to power magnetic fields - epidemiological investigations. Med Pr. 2009; 60(3): 223-233.

10 World Health Organization International Agency for Research on Cancer, Smokeless tobacco and some tobacco-specific N-nitrosamines. IARC Monogr Eval Carcinog. Risks Hum. 2007; 89: $1-592$. 
11 White AJ, O’Brien KM, Niehoff NM, Carroll R, Sandler DP. 2018. Metallic Air Pollutants and Breast Cancer Risk in a Nationwide Cohort Study. Epidemiology; doi:10.1097/EDE.0000000000000917 [Online 06 September 2018].

12 Martin AM, Weber BL. Genetic and hormonal risk factors in breast cancer. J Natl Cancer Inst. 2000;92:1126-1135.

13 Mabuchi K, Bross DS, Kessler II. Risk factors for male breast cancer. J Natl Cancer Inst. 1985;74:371-375.

14 Data were provided by the National Cancer Registration and Analysis Service (part of Public Health England), on request through the Office for Data Release, November 2019.

15 Howlader N, Noone AM, Krapcho M, et al. SEER Cancer Statistics Review, 1975-2016. National Cancer Institute; 2019.

16 Klein SL, Flanagan KL. Sex differences in immune responses. Nat Rev Immunol. 2016; 16: 626- 638.

17 Burstein HJ, Lacchetti C, Anderson H, et al. Adjuvant Endocrine Therapy for Women With Hormone Receptor-Positive Breast Cancer: ASCO Clinical Practice Guideline Focused Update. J Clin Oncol. 2019;37(5):423-438.

18 Conant EF, Barlow WE, Herschorn SD, et al. Association of Digital Breast Tomosynthesis vs Digital Mammography With Cancer Detection and Recall Rates by Age and Breast Density. JAMA Oncol. 2019;28:28 